\title{
Phenolic characterization and antioxidant capacity of ten autochthonous vines grown in southern Italy / Caratterizzazione fenolica e potere antiossidante di dieci vitigni autoctoni allevati nel Sud Italia
}

Rosa Anna Milella ${ }^{1}$, Rosa Pisani ${ }^{2}$, Lisa Mastrofrancesco ${ }^{1}$, Vittorio Alba ${ }^{1}$, Maria Angela Giannandrea ${ }^{1}$, Marica Gasparro ${ }^{1}$, e Angelo Raffaele Caputo ${ }^{1}$

${ }^{1}$ CREA-UTV Consiglio per la ricerca in agricoltura e l'analisi dell'economia agraria - Unità di ricerca per l'uva da tavola e la vitivinicoltura in ambiente mediterraneo, via casa massima 148, 70010 Turi (Ba), Italy

${ }^{2}$ Università degli Studi di Ferrara, Facoltà di Farmacia, 44121 Ferrara, Italy

\begin{abstract}
In plant foods are naturally present some bioactive compounds, that are compounds having or not nutritional value and with biological activity that is expressed in reducing the risk of developing many chronic diseases, therefore leading a key protective effect on our health. Within this group of compounds the antioxidants are included. The importance of antioxidants contained in food is associated with their ability to exert in vivo, in the human body, beneficial effects against chronical- degenerative diseases induced by oxidative stress and age. It has been attributed a positive role to grape polyphenols in terms of increase in endogenous antioxidant defenses, thanks to regulation of genes coding for key enzymes of antioxidant system. For the polyphenols it has also been recognized a specific action of tumor growth inhibition, linked to the modulation of enzymes involved in carcinogenesis or to the inhibition of growth factors and cell proliferation activation. After carbohydrates and acids, the phenolic compounds represent the largest group among grape constituents. The synthesis of these secondary metabolites takes place in two distinct phases of vine growth cycle: fruit set and maturation. The polyphenolic composition contributes to grapes and wine sensory properties, such as color, flavor, astringency, and determines the antioxidant capacity of the extract. These metabolites are mainly related to the variety and their content is influenced by climatic and environmental factors. Among the polyphenols, anthocyanins, hydroxicinnamiltartaric acids, flavonols, flavans, stilbene and resveratrol are of particular interest. Despite numerous studies in the vine-wine industry on polyphenols quantification and qualification, we don't know much about the environmental conditions that affect their synthesis in grapes and how they are extracted from it in wine production. Therefore, the aim of this work has been the study of antioxidant property and phenolic profile of ten autochthonous vines grown in two different areas of South Italy. By spectrophotometric analysis it has been possible to analyze qualiquantitive characteristics of such substances, while by ORAC method (Oxygen Radical Absorbance Capacity) we have measured, in vitro, the antioxidant action. The oenological potential has been evaluated, in relation to polyphenols content, of ten Vitis Vinifera varieties belonging to autochthonous vines of Basilicata, grown in espalier and tent in two areas: in the vineyard of Val d'Agri (PZ), in Basilicata, and in South-East Bari area, in Rutigliano (Ba), in Puglia. The ten varieties belonging to Southern Italy autochthonous vines include four black grapes and six white grapes. Data obtained on total polyphenols content, view of the considerable variability encountered, allow us to affirm that the polyphenolic ripening of wine grapes, thus the reaching of the maximum level, is very influenced by the "terroir", defined as the cultivation area or environment, by season trend, by cultivation techniques and by the different vines nutritional conditions. The same holds true for the antioxidant activity of the 10 wine grapes varieties of this study, since it is closely related to the polyphenol content.
\end{abstract}

Sintesi. Dopo i carboidrati e gli acidi, i composti fenolici rappresentano il gruppo più numeroso tra i costituenti dell'uva. La composizione polifenolica contribuisce alle proprietà sensoriali dell'uva e del vino, quali il colore, il sapore, l'astringenza, e determina il potenziale antiossidante degli estratti. Tali metaboliti sono composti legati principalmente alla varietà, e il loro contenuto è influenzato da fattori climatici ed ambientali. Oggetto di questo lavoro è stato lo studio delle proprietà antiossidanti e del profilo fenolico di dieci vitigni autoctoni allevati in due areali differenti del Sud Italia. Attraverso l'analisi spettrofotometrica è stato possibile analizzare l'aspetto quali e quantitativo delle sostanze, mentre attraverso il metodo ORAC (Oxigen Radical Absorbance Capacity) è stato possibile misurare, in vitro, l'azione antiossidante. I dati ottenuti sul contenuto in polifenoli totali e l'attività antiossidante, vista la notevole variabilità riscontrata, permettono di affermare che la maturazione polifenolica delle uve da vino, quindi, il raggiungimento del livello massimo, è condizionato molto dal "terroir", inteso come zona o ambiente di coltivazione e dall' andamento stagionale, dalle tecniche di coltivazione e, quindi, dalle diverse condizioni nutrizionali delle viti. 


\section{Introduzione}

L'uva, il vino e i sottoprodotti dell'industria enologica sono fonti ricche di composti polifenolici tra cui flavonoli, antociani, flavanoli e proantocianidine. Questi composti svolgono un ruolo importante non solo nella qualità delle uve e dei vini in quanto determinano le caratteristiche sensoriali, come il colore, il sapore e l'astringenza, ma anche per i loro effetti benefici sulla salute umana. Sono, infatti, componenti fondamentali della dieta umana per la loro dimostrata attività antiossidante, la capacità di catturare i radicali liberi e mitigare lo stress ossidativo prodotto nel corso di malattie croniche e degenerative [1].

I composti polifenolici dell'uva possiedono importanti attività biologiche: antiossidanti [2]; protettive del sistema cardiocircolatorio [3,4]; anticancro [5]; antinfiammatorie [6]; anti-età [7]; antimicrobiche [8].

L'attività antiossidante rappresenta una delle più importanti proprietà dei composti fenolici dell'uva e del vino. I polifenoli dell'uva sono importanti antiossidanti a funzione multiple: possono agire come agenti riducenti, antiossidanti donatori di idrogeno o agenti chelanti dei cationi metallici. Indirettamente, i polifenoli possono interferire con i sistemi di detossificazione cellulare, come la superossido dismutasi (SOD), la catalasi o la glutatione perossidasi [9]. Infine, i flavonoidi possono inibire enzimi che producono ROS (Reactive Oxygen Species) come la xantina ossidasi e la nicotinamide adenina dinucleotide fosfato ossidasi (NADPH) $[10,11]$.

\section{Materiali e metodi}

\subsection{Uve utilizzate per lo studio e ambienti di coltivazione}

Le analisi sono state effettuate sulle uve dei seguenti vitigni locali e/o autoctoni minori: I - vitigni a bacca nera: a) Aglianico dolce precoce n., b) Vuianese n., c) Primitivo (Viggiano) n., d) Nerona (Viggiano) n.; II - vitigni a bacca bianca: e) Iuvino b., f) Guarnaccia b., g) Malvasia bianca di Lucania b. (biotipo 1), h) Malvasia bianca di Lucania b. (biotipo 2), i) Malvasia bianca (Maccarrone) b., 1) Trebbiano antico b. (Fig. 1).

Le uve analizzate per la prova provengono da due vigneti coltivati in due differenti regioni del Sud d'Italia: in Basilicata e in Puglia. Il primo vigneto sperimentale è ubicato nel Comune di Marsicovetere in provincia di Potenza, presso l'Azienda Agricola Sperimentale Dimostrativa (A.A.S.D.) "Bosco Galdo" di Villa d'Agri dell'Agenzia Lucana di Sviluppo e Innovazione in Agricoltura (A.L.S.I.A.) della Regione Basilicata. Il secondo vigneto sperimentale fa parte della collezione ampelografica presso l'azienda sperimentale "Lamarossa" del CREA-UTV che si trova in agro di Rutigliano, in provincia di Bari.

In genere il clima della Basilicata è mediterraneo lungo le coste, ma ha chiare caratteristiche continentali procedendo verso l'interno e sui rilievi maggiori ha le caratteristiche tipiche del clima di montagna. Passando allo specifico della zona interessata, la Val d'Agri ha un clima che si caratterizza per un moderato deficit idrico estivo e per una concentrazione estiva dell'efficienza termica.

Per quanto concerne il secondo vigneto sperimentale, esso è situato in Puglia ed esattamente in agro di Rutigliano, un comune che si trova nel sud-est barese. Il

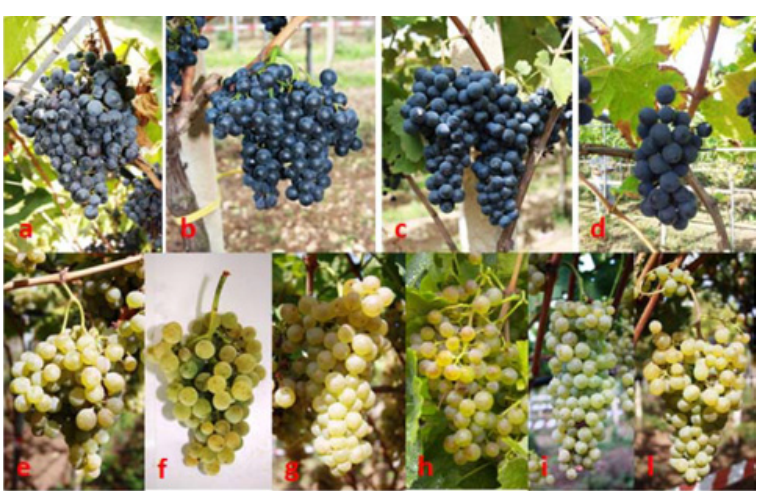

Figura 1. I vitigni oggetto d'esame.

sito dove insiste il vigneto sperimentale è caratterizzato da condizioni pedo-climatiche ideali per lo sviluppo vegetoproduttivo della vite con terreni di medio impasto ricchi di potassio e di calcio, luminosità elevata, clima mite anche di inverno, caratterizzato da discreta piovosità nel periodo invernale e da scarse precipitazioni in quello primaverileestivo.

\subsection{Estrazione polifenoli dalle bucce e dai vinaccioli}

Le analisi chimico-analitiche sono state eseguite su campioni di grappoli, raccolti a maturità sul terzo mediano dei capi a frutto, nel corso dell'annata 2014. Dai grappoli raccolti, dalla parte centrale sono stati prelevati 30 acini, selezionati a random. I 30 acini sono stati pesati, lavati e successivamente pelati. Le bucce e i vinaccioli sono stati immersi in $25 \mathrm{~mL}$ di una soluzione di etanolo cloridrico (70:30:1/acqua:etanolo:acido cloridrico) e lasciati in infusione per una notte. L'estratto è stato separato dalle componenti solide per decantazione, filtrato con filtri di cellulosa rigenerata da $0,45 \mathrm{~mm}$ e conservati a $-20^{\circ} \mathrm{C}$.

\subsection{Analisi polifenoli in spettrofotometria}

Il contenuto polifenolico totale di ciascun estratto è stato determinato con il metodo colorimetrico di Folin Ciocalteau in microscala [12]. Nel lavoro abbiamo utilizzato $1 \mathrm{~mL}$ di acqua, $0,02 \mathrm{~mL}$ di estratto, $0,2 \mathrm{~mL}$ di reagente di Folin-Ciocalteau e $0,8 \mathrm{~mL}$ di carbonato di sodio al $10 \%$, miscelati e portati a $4 \mathrm{~mL}$ con acqua distillata. Dopo 90 minuti di incubazione è stata registrata l'assorbanza a $765 \mathrm{~nm}$ e i risultati espressi in peso fresco (F.W.) di uva $(\mathrm{mg} / \mathrm{Kg})$ come equivalenti di ac. gallico (GAE).

Il contenuto antocianico totale è stato misurato diluendo gli estratti con il solvente usato per l'estrazione e leggendo l'assorbanza a $540 \mathrm{~nm}$. Il valore ottenuto è stato moltiplicato per il fattore di diluizione e un opportuno coefficiente di conversione $(26,6)$ valido per la stima degli antociani totali [13].

\subsection{Determinazione dell'attività anti-ossidante con il metodo ORAC}

Per la determinazione dell'attività antiossidante degli estratti di bucce e semi è stato utilizzato il test ORAC (Oxygen Radical Absorbance Capacity) [14]. In breve, in 


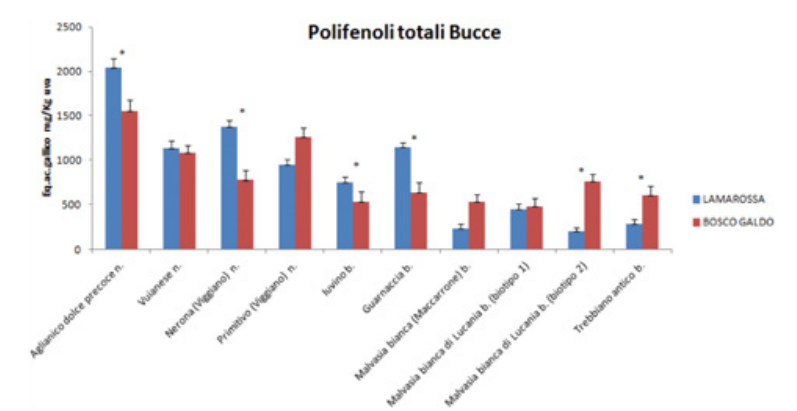

Figura 2. Contenuto polifenolico totale delle bucce di 10 varietà di uva da uva da vino allevate nei due ambienti di "Bosco Galdo" di Villa d'Agri (PZ) e di "Lamarossa" di Rutigliano (BA). I valori sono espressi in equivalenti di ac. gallico ( $\mathrm{mg} / \mathrm{Kg}$ di uva fresca). Ogni valore è la media di tre misurazioni. $\left({ }^{*} \mathrm{p}<0,05\right)$.

micropiastre da 96 pozzetti, sono stati aggiunti $150 \mathrm{uL}$ di fluoresceina $(7,7 \mu \mathrm{M}), 25 \mu \mathrm{L}$ di campione opportunamente diluito e $25 \mu \mathrm{L}$ di AAPH $(221,3 \mathrm{mM})$. La stessa procedura è stata utilizzata per ottenere la retta di calibrazione con il Trolox usato come standard. La fluorescenza, misurata a $\lambda$ exc $=485$ e $\lambda$ ems $=535$ con fluorimetro FLUORstar OPTIMA (BMG LABTECH), è stata registrata ogni $90 \mathrm{sec}$. per 90 minuti. I risultati sono stati calcolati usando la differenza delle aree sottese sotto le curve di decadimento della fluoresceina, ottenute in presenza ed in assenza del campione (AUC) e sono espressi come $\mu \mathrm{MTrolox}$.

\subsection{Analisi statistica}

I campioni sono stati preparati e analizzati in triplicato. Per ogni campione sono stati riportati media e deviazione standard (SD). I risultati sono stati elaborati utilizzando il t-test. Sono state considerate significative differenze con $\mathrm{p}<0,05$.

\section{Risultati e discussione}

\subsection{Polifenoli totali}

Nel lavoro sono stati quantificati i polifenoli totali presenti nelle bucce e nei semi di 10 varietà di uva da vino allevate nei due ambienti di Villa d'Agri (PZ), azienda "Bosco Galdo", e di Rutigliano (BA), azienda "Lamarossa". Il contenuto di polifenoli totali nelle bucce delle 10 varietà di uva da vino prese in esame varia da un valore minimo di 208 eq. Acido gallico mg/Kg di uva della Malvasia bianca di Lucania b. (biotipo 2) ad un valore massimo di 2.047 eq. Acido gallico $\mathrm{mg} / \mathrm{Kg}$ dell'Aglianico dolce precoce $\mathrm{n}$. (Fig. 2).

Le varietà bianche sono quelle che, generalmente, hanno un più basso contenuto polifenolico poiché non possiedono la classe degli antociani.

Per quanto riguarda i semi, come si evince dalla Fig. 3, il range di variabilità va da 139 della Nerona (Viggiano) n. a 1.756 eq. Acido gallico $\mathrm{mg} / \mathrm{Kg}$ dell'Aglianico dolce precoce $n$.

L'andamento dei polifenoli totali riscontrato nelle bucce per le due diverse località viene confermato anche nei semi. I valori riscontrati nelle bucce e nei semi sono paragonabili, nonostante dalla letteratura è noto che nei semi i livelli di polifenoli sono più elevati; tale andamento si spiega in quanto, in questo studio, i semi non sono stati frantumati.

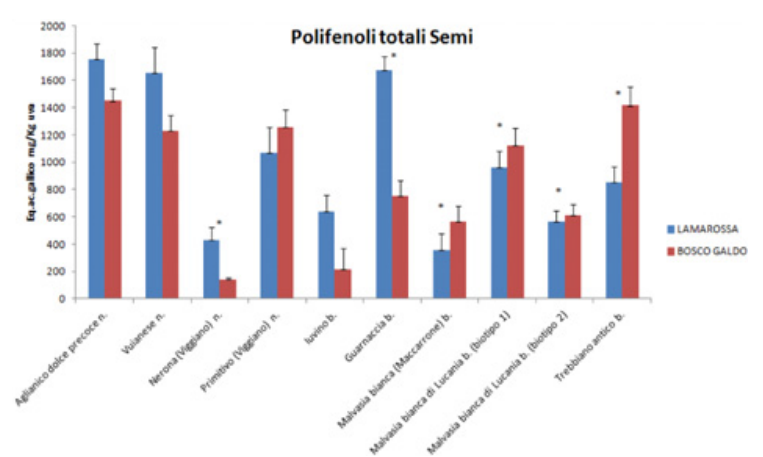

Figura 3. Contenuto polifenolico totale dei semi di 10 varietà di uva da uva da vino allevate nei due ambienti di "Bosco Galdo" di Villa d'Agri (PZ) e di "Lamarossa" di Rutigliano (BA). I valori sono espressi in equivalenti di ac. gallico $(\mathrm{mg} / \mathrm{Kg}$ di uva fresca). Ogni valore è la media di tre misurazioni $\left({ }^{*} \mathrm{p}<0,05\right)$.

Analizzando le varietà distinte in due gruppi per colore della bacca, si può notare che i valori dei primi tre vitigni a bacca nera (Aglianico dolce precoce, Vuianese e Nerona) riscontrati nel campo sperimentale di "Lamarossa" sono superiori a quelli di "Bosco Galdo". Di questi vitigni, soltanto il Primitivo coltivato a "Bosco Galdo" è risultato superiore a quello coltivato a "Lamarossa"; va comunque precisato che i valori riscontrati di quest'ultimo vitigno non sono risultati significativamente differenti (942 di "Lamarossa" contro 1.260 eq. Acido gallico mg/Kg di "Bosco Galdo", per le bucce; 1.069 di "Lamarossa" contro 1.258 eq. Acido gallico mg/Kg di "Bosco Galdo", per i semi).

Nel gruppo dei vitigni a bacca nera, le uniche differenze significative sono state registrate per i valori delle bucce nel vitigno Aglianico dolce precoce, che ha fatto registrare un valore di 2.047 di "Lamarossa" contro un valore inferiore di 1.548 eq. Acido gallico $\mathrm{mg} / \mathrm{Kg}$ di "Bosco Galdo"; mentre, per la Nerona entrambi i valori delle bucce e dei semi sono risultati superiori nell'uva coltivata a "Lamarossa" rispetto a quella coltivata a "Bosco Galdo": infatti, abbiamo riscontrato per le bucce 1.375 contro 774 eq. Acido gallico mg/Kg e, per i semi, 431 contro 139 eq. Acido gallico $\mathrm{mg} / \mathrm{Kg}$. Per i vitigni a bacca bianca, la tendenza si inverte; infatti, su sei varietà prese in considerazione, quattro varietà hanno fatto registrare valori di polifenoli più alti per l'ambiente lucano. Le quattro varietà dell'ambiente lucano che hanno fatto registrare $i$ valori più alti sono state: Malvasia bianca (Maccarrone), Malvasia bianca di Lucania (biotipo 1 e 2) e Trebbiano antico. Di queste quattro varietà, soltanto in due varietà, Malvasia bianca di Lucania (biotipo 2) e Trebbiano antico sono risultati significativamente differenti sia i valori delle bucce sia i valori dei semi; mentre, con riferimento ai semi tutte e quattro le varietà hanno fatto registrare valori significativamente più alti delle uve coltivate a "Bosco Galdo" contro quelle coltivate a "Lamarossa": nell'ordine abbiamo che in ambiente lucano la Malvasia bianca (Maccarrone) ha fatto registrare 566, la Malvasia bianca di Lucania (biotipo 1) 1.123, la Malvasia bianca di Lucania (biotipo 2) 615 e il Trebbiano antico 1.415 eq. Acido gallico mg/Kg, contro i 356, 961, 565 e 853, rispettivamente per l'ambiente pugliese. Al riguardo va sottolineato che delle 10 varietà sotto esame, sono da considerarsi 'prettamente autoctoni' proprio questi quattro 


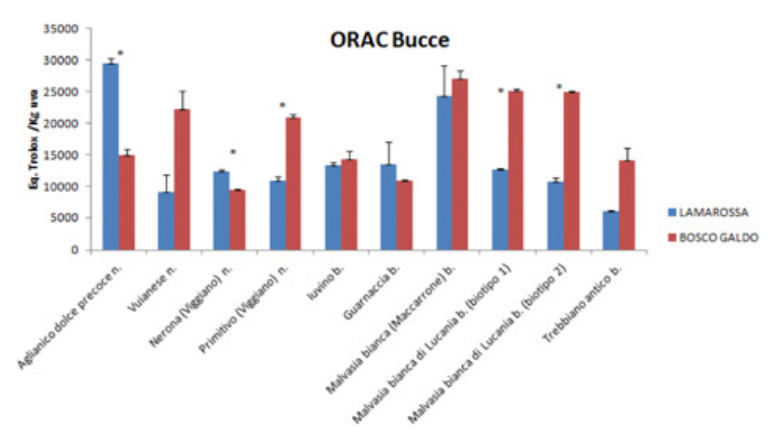

Figura 4. Attività anti-ossidante delle bucce di uve da vino allevate nei due ambienti di "Bosco Galdo" di Villa d'Agri (PZ) e di "Lamarossa" di Rutigliano (BA) valutata con il metodo ORAC. I risultati sono espressi in micromoli di Trolox equivalenti per $\mathrm{Kg}$ di peso fresco di uva ( $\mu$ mol TE/Kg di peso fresco). Ogni valore è la media di tre misurazioni $(* \mathrm{p}<0,05)$.

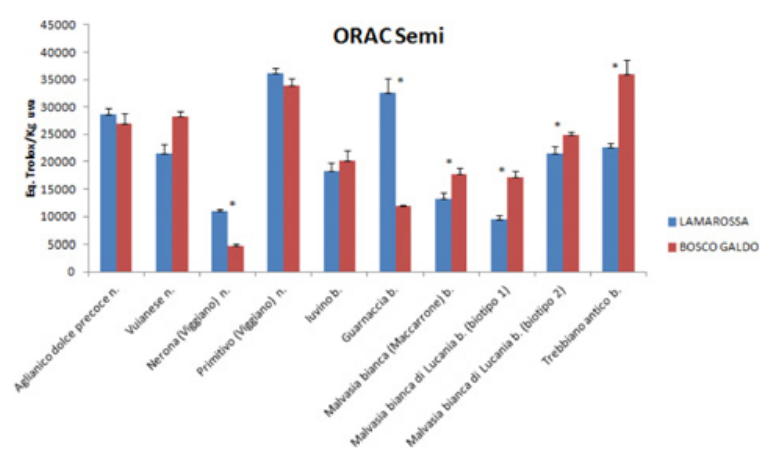

Figura 5. Attività anti-ossidante dei semi di uve da vino allevate nei due ambienti di "Bosco Galdo" di Villa d'Agri (PZ) e di "Lamarossa" di Rutigliano (BA) valutata con il metodo ORAC. I risultati sono espressi in micromoli di Trolox equivalenti per $\mathrm{Kg}$ di peso fresco di uva ( $\mu$ mol TE/Kg di peso fresco). Ogni valore è la media di tre misurazioni $\left({ }^{*} \mathrm{p}<0,05\right)$.

vitigni. Infatti, mentre le altre denominazioni in vernacolo utilizzate sono riconducibili a denominazioni varietali ufficialmente note nel Registro Nazionale delle Varietà di Vite, la cui diffusione è nota anche in altre regioni viticole, le denominazioni in vernacolo di Malvasia bianca (Maccarrone), Malvasia bianca di Lucania (biotipo 1 e 2) e Trebbiano antico si riferiscono a vitigni del tutto originari del territorio lucano, come richiamato dalle stesse denominazioni.

\subsection{Unità ORAC}

L'attività antiossidante degli estratti preparati dalle bucce e dai semi delle 10 varietà di uva da vino è stata valutata con il metodo ORAC (Oxygen Radical Absorbance Capacity). I risultati sono mostrati nelle Figs. 4 e 5 dalle quali si evince, un andamento più o meno similare a quello visto per i polifenoli, a dimostrazione di una significativa correlazione fra contenuto polifenolico delle uve e unità ORAC.

Il valore minimo riscontrato di 6.061 eq. Trolox $/ \mathrm{Kg}$ di uva peso fresco (Fresh Weight) è del Trebbiano antico b., mentre l'Aglianico dolce precoce n. possiede il valore massimo di 29.539 eq. Trolox/Kg F.W., entrambi coltivati nella stessa località pugliese ("Lamarossa" di Rutigliano).

Prendendo in considerazione soltanto le varietà con valori che differiscono significativamente, sia per le

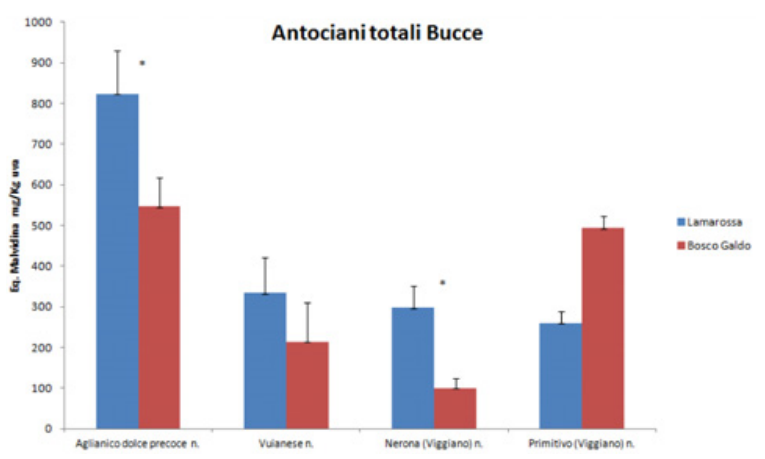

Figura 6. Antociani totali delle bucce di 10 varietà di uva da vino allevate nei due ambienti di "Bosco Galdo" di Villa d'Agri (PZ) e di "Lamarossa" di Rutigliano (BA). I valori sono espressi in equivalenti di malvidina $(\mathrm{mv})$ per $\mathrm{Kg}$ di uva fresca $(\mathrm{mg}$ di $\mathrm{mv} / \mathrm{Kg}$ peso fresco). Ogni valore è la media di tre misurazioni $\left({ }^{*} \mathrm{p}<0,05\right)$.

bucce che per i semi, notiamo che anche nel caso dell'attività antiossidante i vitigni cosiddetti 'prettamente autoctoni', coltivati nel loro ambiente lucano di origine, sono quelli che presentano elevata attività antiossidante rispetto, ovviamente, agli stessi coltivati in diverso ambiente. Infatti, per le bucce, ritroviamo che la Malvasia bianca (Maccarrone), i due biotipi di Malvasia bianca di Lucania e il Trebbiano antico coltivati a "Bosco Galdo" (27.029, 25.026, 24.881 e 14.179 eq. Trolox/Kg F.W., rispettivamente) presentano valori più elevati di unità ORAC di quelli coltivati a "Lamarossa" (24.318, 12.603, 10.680 e 6.061 eq. Trolox/Kg F.W., rispettivamente), con differenze significative tra gli ultimi tre valori; eccezion fatta per i valori della Malvasia bianca (Maccarrone).

Sono, invece, tutte e quattro significative le differenze tra i valori relativi ai semi; infatti, abbiamo che la Malvasia bianca (Maccarrone), i due biotipi di Malvasia bianca di Lucania e il Trebbiano antico coltivati a "Bosco Galdo" hanno espresso 17.732, 17.115, 24.830 e 35.991 eq. Trolox/Kg F.W., rispettivamente, contro 13.227, 9.469, 21.380 e 22.655 eq. Trolox/Kg F.W., degli stessi coltivati a "Lamarossa".

\subsection{Antociani totali}

Per questa classe di composti fenolici, presenti soltanto nei vitigni a bacca nera, in termini di valori riscontrati nelle bucce, è opportuno segnalare una certa correlazione tra il contenuto in Antociani totali e Unità ORAC. Infatti, con riferimento solamente alle differenze significative, come si evince dalla Fig. 6, i vitigni che hanno presentato valori più elevati di Antociani totali sono stati l'Aglianico dolce precoce coltivato a "Lamarossa" con 824 eq. Malvidina $\mathrm{mg} / \mathrm{Kg}$ di uva contro 546 dello stesso vitigno coltivato a "Bosco Galdo"; la Nerona (Viggiano) con 297 di "Lamarossa" contro 100 eq. Malvidina mg/Kg di uva di "Bosco Galdo"; e invertendo la situazione, abbiamo il Primitivo (Viggiano) con 493 di "Bosco Galdo" contro 259 eq. Malvidina $\mathrm{mg} / \mathrm{Kg}$ di uva di "Lamarossa".

Lo stesso andamento è stato registrato per l'attività antiossidante; infatti, ritornando alla Fig. 3, l'Aglianico dolce precoce e la Nerona (Viggiano) coltivati a "Lamarossa" hanno fatto registrare, rispettivamente, valori di 29.539 e di 12.357 eq. Trolox/Kg F.W. più elevati degli stessi vitigni coltivati a "Bosco Galdo", dove, invece, 
hanno fatto registrare valori di 14.951 e di 9.425 eq. Trolox/Kg F.W.; per il Primitivo (Viggiano) la situazione è invertita, con 20.845 eq. Trolox/Kg F.W. di "Bosco Galdo" rispetto a 10.873 eq. Trolox/Kg F.W. di "Lamarossa".

\section{Conclusioni}

Questo studio, in linea con gli altri lavori presenti in letteratura, conferma che l'uva è un'importante fonte alimentare di antiossidanti naturali per la prevenzione di malattie causate dallo stress ossidativo. Come già noto, non tutte le varietà sono uguali ma i caratteri genetici (genotipo) influenzano notevolmente la qualità dell'uva. Tuttavia, esaminando la stessa varietà di vitigni allevata in due ambienti diversi, è possibile affermare che la maturazione polifenolica delle uve da vino è condizionata molto dal "terroir", inteso come zona o ambiente di coltivazione e dall' andamento stagionale, dalle tecniche di coltivazione, e quindi dalle diverse condizioni nutrizionali delle viti. Sia i dati ottenuti sul contenuto in polifenoli totali che quelli per l'attività antiossidante delle 10 varietà di uva da vino, oggetto di questo studio, esprimono una notevole variabilità tra $\mathrm{i}$ due ambienti.

Tuttavia, relativamente al genotipo, è possibile avanzare una considerazione conclusiva. Da questo studio emerge che i vitigni cosiddetti 'prettamente autoctoni', quali la Malvasia bianca (Maccarrone) b., i due biotipi di Malvasia bianca di Lucania (o di Basilicata) b. e il Trebbiano antico b. quando sono coltivati nel loro ambiente lucano di origine esprimono maggiori livelli di contenuto polifenolico e quindi una migliore performace fisiologica (maturazione polifenolica).

Inoltre, la caratterizzazione della biodiversità, sia in questo lavoro che in tanti altri studi, permette di valorizzare le varietà che meglio si adattano a un ambiente arricchendosi di sostanze nutraceutiche importanti per la salute umana.

\section{Riferimenti}

[1] K.R. Martin, C.L. Appel. Nutr. Diet. Suppl 2: 1-12 (2010)

[2] E. Xia, G. Deng, Y. Guo, H. Li . Int. J. Mol. Sci., 11 $2(2010)$

[3] R.A. Milella, D. Antonacci, P. Crupi, F. Incampo, C. Carrieri, N. Semeraro, M. Colucci. J. Food Sci. 77, 8 (2012)

[4] C. Carrieri, R.A. Milella, F. Incampo, P. Crupi, D. Antonacci, N. Semeraro, M. Colucci. Food Chem. 140, 647-653 (2013)

[5] J. Yu, M. Ahmedna. Int. J. Food Sci. Technol. 48, 2 (2013)

[6] L. Wang, G.D. Stoner. Cancer Lett. 269, 2 (2008)

[7] F. Zhang, J. Liu, J.S. Shi. Eur. J. Pharm. 636, 1-7 (2010)

[8] G. Simonetti, A.R. Santamaria, F.D. D'Auria, N. Mulinacci, M. Innocenti, F. Cecchini, E. Gabrielli, S. Panella, D. Antonacci, A.T. Palamara, A. Vecchiarelli, G. Pasqua. BioMed Research International (2014)

[9] O.L. Woodman and E. Chan. Clin Exp Pharmacol Physiol 31, 11 (2004)

[10] R.J. Nijveldt, E. van Nood, D.E. van Hoorn, P.G. Boelens, K. van Norren and P.A. van Leeuwen. Am J Clin Nutr 74, 4 (2001)

[11] F. Orallo, E. Alvarez, M. Camina, J.M. Leiro, E. Gomez and P. Fernandez. Mol Pharmacol 61, 2 (2002)

[12] A.L. Waterhouse. In R.E. Wrolstad (Ed.) New York: John Wiley \& Sons Inc (2009)

[13] E. Di Stefano, M.C. Cravero. Rivista di Viticoltura e di Enologia. 2, 37-45 (2001)

[14] B. Ou, M. Hampsch-Woodill, Prior R.L. Journal of Agricultural and Food Chemistry 49, 4619-4626 (2001) 